\title{
Discussion on Backup and Recovery Technology of Computer Database
}

\author{
Xiangrong ZHOU \\ Information engineering college, fujian meizhouwan Career Technical College \\ Putian 351100,China .
}

\begin{abstract}
In the process of social and economic development in our country, China's science and technology is also constantly improved, including computer network technology. The emergence of network technology provides convenience for people's work and life, but there are many problems in the use of network technology. For example, due to a variety of factors, resulting in the computer database information will be destroyed, in order to ensure the stability and security of the data and data in the computer database, the database backup and recovery technology should be studied deeply. Based on this, this paper discusses the backup and recovery technology of computer database, and puts forward the advanced technology of computer database backup and recovery, so as to ensure the security of data and data in computer database and reduce the risk of computer database.
\end{abstract}

Keywords: Database; backup; recovery

\section{Introduction}

Computer network technology has an important role in people nowadays life, social and modern technology development are inseparable from the computer network technology. Computer database backup and recovery technology is the computer in the process of running the most basic and most important technology, the computer database backup technology to backup and copy the computer system data and information, save the data and information. Computer recovery technology can retrieve the computer system lost data and information to avoid the reasons for the computer system damage, resulting in data loss, can retrieve the data information.

\section{The computer database}

The database is stored in a certain way, can achieve multi-user sharing, with the characteristics of small redundancy, and computer programs work independently of each other. In short, the computer database is an electronic file cabinet, used to store information and books in the computer, the user can add data to the file, update, delete and other operations. The data in the database are created for the majority of users to create information sharing, out of the specific procedures of the constraints and restrictions, different users can use their own way to use the database of information and data, and multiple users can achieve data in the database Sharing, data sharing can effectively meet the needs of users of data information, and also meet the data communication between different users. ${ }^{\text {[11 }}$

Computer database is divided into three results, they reflect the database from different angles, respectively, for the data physical layer, the concept of data layer, the user data layer. The physical data layer refers to the innermost layer of the database, its main role is the physical storage device data, these data are all raw data, but also the user needs to process the object, which is mainly through the internal mode description of the instruction characters, String and word composition; The conceptual data layer refers to the middle layer of the database, which is the logical representation of the database as a whole. The concept data layer indicates the logical definition of each data and the logical relationship between the different data, and can also store and record these links and definitions. To the database for all the logical relationship between the object, not the physical situation; The user data layer refers to the database that the user can see and use, which primarily represents the set of data used by one or some specific users, that is, a logical collection. The links at each level of the database are converted by mapping. 


\section{The computer database backup technology}

With the continuous development and improvement of computer database backup technology, the computer due to the failure of the data loss caused by the probability is constantly reduced, the computer running in the process of various problems and obstacles, which are associated with the corresponding preventive measures. In short, the computer to store information on the effective backup and integration of the entire process is the implementation of computer backup technology process. Computer database in the backup process mainly includes two forms, namely, static backup and dynamic backup. In the process of dynamic backup, the integrity and timeliness of the data cannot be well protected; In the process of static backup data integrity and effectiveness can be effectively protected, and if the data loss during the backup process, but also as soon as possible to restore.

\subsection{The database backup form and characteristics,}

Database backup, including dynamic backup and static backup, dynamic backup features: in the process of database backup, the database can be modified and access instructions, that is, the database backup and user activities can be carried out together; Static backup is characterized by: in the process of database backup, the database cannot be modified and access instructions. In general, the static backup of the database is relatively simple, but the static backup has a large limitation, only in the users other activities can be completed after the backup, so this backup can reduce the availability of the database. Database dynamic backup and not the above shortcomings, which can be carried out with the activities of users, database dynamic backup of the limitations are: in the realization of the backup, the computer does not guarantee the copy of the data in the correctness and effectiveness. ${ }^{\text {[31 }}$

For example, in a certain period of time to the computer database to perform a dynamic backup, the data copied to other disk, but in the next time to modify the data to be backed up, so after the end of the backup, the copy of the data is not modified the data. So, when we are a dynamic backup of the database, to record the database in the backup process of all the changes, and record the action created as a log file, then the disk backup copy and log files of each other, you can meet The database is backed up in the correct state for a certain period of time.

\subsection{The database backup technology}

\subsubsection{SAN backup technology}

Database SAN backup technology refers to the use of high-performance optical connection to the computer in the server and disk, and then through the data administrator on the computer storage system for centralized management, and the use of high-speed optical to achieve data transmission, but also according to the user The different needs of the use of different ways to access, so that the data can be shared storage. ${ }^{\mathbf{4} \mathbf{I}}$

\subsubsection{Classification and archiving of backups}

The main advantages of database grading and archiving are the ability to integrate data information in an orderly manner, enabling the specification and cleanup of large amounts of information in the database to provide more storage space for data in the computer database. Data archiving refers to the realization of data copy and copy, the main purpose is to ensure that the computer data in the security of information, and make it long-term storage. 


\subsubsection{Network backup}

The network backup of the database mainly refers to the transfer of data information to the data host to be backed up, and then uses the computer network data transfer function to transfer the data to other servers.

This data backup data can be directly connected with the server, as a result of data backup brings a variety of risk factors, increasing the burden on the server. To be able to effectively solve this problem, we must back up through the data network backup data backup, in order to improve the efficiency and security of network backup.

\subsubsection{High performance backup}

High-performance backup of the database refers to the high-performance data through the software to achieve the conversion of database information. If the performance of the general data software, the computer data information is large, the software information backup conversion rate will continue to decline, and even may lead to the collapse of the system, so we must use a high performance data software, In order to improve the security and speed of data conversion. ${ }^{\mathbf{5} \mathbf{1}}$

\subsubsection{Remote backup}

As the computer database stored information will be affected by various factors outside the world, such as human tampering, virus intrusion, etc., these problems must be restored through conventional data backup technology, if you encounter natural disasters, it is necessary to remote backup To achieve the backup of data, through computer software or hardware systems will be business data information into a remote backup system, in order to avoid the natural disasters on the data caused by damage. IN the process, the relevant staff should pay close attention to the local data field remote data check.

\section{The computer database recovery technology}

In our daily work or learning process, will often appear computer database failure. For example, in the computer work during the process of virus intrusion, system crashes, battery failure and so on. These failures and problems will pose a threat to the normal use of the computer, but also seriously affect the computer database information security and integrity. As can be seen, the computer database recovery technology has an important role. ${ }^{\mathbf{I} 6 \mathbf{1}}$

\subsection{The database operation}

Computer database in the process of running there will be power interruption, viruses and disk damage and other issues, resulting in data loss or damage in the database, resulting in paralysis of the database phenomenon, improve the work pressure. Therefore, the relevant personnel will be on the computer database recovery technology attention. Database recovery methods and principles is based on the creation of a backup log, once the database fails, mainly including two cases: First, the database did not suffer damage, but some parts of the database found abnormal. For example, after the program is modified suddenly interrupt, if this happens, first of all through the log database to revoke the order, then the database will be able to return to the normal state; Second, the database has been severely damaged, so that the disk has been damaged, resulting in the disk cannot be used. This time will be the final copy of the database saved to another disk, as a result through the log database recovery. 


\subsection{Checkpoint technology}

If the computer database fails, to restore the subsystem, through the log database to judge, but this process is very slow, and there are many processes have been modified in the disk, then re-do it will extend the recovery process. IN order to be able to effectively improve the efficiency of the log database, you can use checkpoint technology. The In the process of running the database, the system to make the log and cycle checkpoint, in the checkpoint, the first memory in the memory and output to a stable memory; In addition to the database buffer has been modified after the contents of the transfer to the disk; Second, to record the checkpoint; finally, the log checkpoint to fill in the record address to restart. The records of the checkpoints to include all the activities in the checkpoint and the nearest activity address, restart the time from the recovery subsystem to get the checkpoint address, from the log to find the checkpoint of the record content, in order to get what activities can be revoked. ${ }^{\text {[71 }}$

\subsection{The overall recovery}

Full recovery has a greater difficulty, the computer database system to restore the overall system is a big problem or change when the time, such as geology, fire and other force majeure factors, in order to complete recovery of the computer database system.

\section{A single recovery}

The single recovery of the database system refers to the restoration of the document through the development of the document is a more common database recovery technology. Single recovery technology is relatively simple, as long as the system backup, query the database system, in order to be able to achieve file recovery. ${ }^{[8]}$

\section{Conclusion.}

All in all, computer databases play an important role in computer systems, not only as a major component of a computer system, but also as a major part of a computer's storage of information. IN the process of continuous progress of science and technology, the database has been widely used in people's lives and work in all aspects of the database backup and recovery technology is also in the community in an irreplaceable position.

\section{References}

[1] Hou W, Liu Y, Lin L, et al. Discussion on Strategy Analysis and Application of Data Redundancy Backup and Disaster Recovery System in Broadcasting Industry[J]. Radio \& Tv Broadcast Engineering, 2015:31-39.

[2]Zhang Y U. On the Main Backup and Recovery Technology of Computer Database[J]. Wireless Internet Technology, 2015:51-58.

[3]Bagchi K, Kapoor A, Chockalingam E, et al. Method and system for a database management system add-in for third party backup and restore applications[J]. 2015:102-115.

[4]Balcha M R. Method and apparatus of managing application workloads on backup and recovery system[J]. 2015:97-106.

[5]Wang F. Elementary Discussion on Deformation Joint Recovery Technology of Large Drainage Culvert[J]. Urban Roads Bridges \& Flood Control, 2016:71-82.

[6]Mittal K, Chavan M, French B, et al. Synchronized backup and recovery of database systems[J]. 2016:11-16. 
[7]Chen J J, Garin B E, Girkar M B, et al. Zero and near-zero data loss database backup and recovery[J]. 2016:15-29.

[8]Parab N. Cloud-based disaster recovery of backup data and metadata[J]. 2016:(10):5-12. 